\title{
STELLAR PULSATIONS AND GRAVITATIONAL WAVES
}

\author{
KOSTAS D. KOKKOTAS \\ Max-Planck-Institut für Gravitationsphysik, Albert-Einstein-Institut \\ D-14473 Potsdam, Germany \\ Max-Planck-Society, Research-Unit Theory of Gravitation, Jena D-07743, Germany \\ E-mail: kokkotas@astro.auth.gr
}

1. Introduction. Pulsating stars are important sources of information for astrophysics. Nearly every star undergoes some kind of pulsation from the early stages of its formation until the very late ones i.e. the catastrophic creation of a supercompact object (white dwarf, neutron star or black hole). Pulsations of supercompact objects are of great importance for relativistic astrophysics since these pulsations are accompanied by the emission of gravitational radiation. In this review we shall discuss various features of neutron star pulsations i.e. the various modes of pulsation, mode excitation, detection probability and the possibility to extract information (to estimate, for example, the radius, mass and stellar equation of state) from the detection of the associated gravitational waves.

2. Excitation mechanisms. It is well known in astrophysics that many stars will end their lives with a violent supernova explosion. This will leave behind a compact object which will oscillate violently in the few first seconds. Huge amounts of gravitational radiation will be emitted and the initial oscillations will consequently damp out. The gravitational waves will carry away information about the compact object. If the supernova remnant is a black hole we will observe a short monochromatic burst lasting a few tenths of a millisecond, while if it is a neutron star we will observe a more complicated signal which will be the overlapping of several frequencies. The stellar signal will consist of a short burst (similar to that from a black hole) followed by a long lasting sinusoidal wave. The characteristics of such a signal will be discussed in the following chapters. Supernovae are quite frequent, the expected event rate is $5.8 \pm 2.4$ events per century per galaxy [30]. The amount of energy emitted in such an event depends on the details of the collapse. A spherical collapse will not produce any gravitational waves at all, while as much as

1991 Mathematics Subject Classification: 83C35, 85A15.

The paper is in final form and no version of it will be published elsewhere. 
$10^{-3} M_{\odot} c^{2}$ can be radiated away in a highly non-spherical one. The present numerical codes used to simulate collapse predict that the energy emitted as gravitational waves will be of the order of $10^{-4}-10^{-6} M_{\odot} c^{2}$ [8]. However, most of these codes are based on Newtonian dynamics and the few fully general relativistic ones are not 3-dimensional. For example, most of the codes fail to explain the high average pulsar velocity which is believed to be a result of a boost that the neutron star gets during the collapse due to anisotropy in the neutrino distribution [10].

Although collapse may be the most frequent source for excitation of stellar oscillations there are other situations in which significant pulsations take place. For example, after the merger of two coalescing neutron stars it is natural to expect that the final object will oscillate. Thus the well known waveform for coalescing binaries will be followed by the characteristic signal of the newly created compact object. In the same way smaller bodies falling on a neutron star or black hole will excite oscillations, and oscillations on a neutron star can be excited by a close encounter with another star.

To obtain rough estimates for the typical gravitational-wave amplitudes from a pulsating star we use the standard relation for the gravitational-wave flux [26]

$$
F=\frac{c^{3}}{16 \pi G}|\dot{h}|^{2}=\frac{1}{4 \pi r^{2}} \frac{d E}{d t},
$$

which is valid far away from the star. Combining this with i) $d E / d t=E / 2 \tau$ where $\tau$ is the e-folding time of the pulsation and $E$ is the available energy ii) the assumption that the signal is monochromatic (with frequency $f$ ) and iii) the knowledge that the effective amplitude achievable after matched filtering scales as the square-root of the number of observed cycles: $h_{\text {eff }}=h \sqrt{n}=h \sqrt{f \tau}$, we get the estimates

$$
h_{\mathrm{eff}} \sim 3 \times 10^{-21}\left(\frac{E}{10^{-6} M_{\odot} c^{2}}\right)^{1 / 2}\left(\frac{2 \mathrm{kHz}}{f}\right)^{1 / 2}\left(\frac{50 \mathrm{kpc}}{r}\right),
$$

for the $f$-mode, and

$$
h_{\mathrm{eff}} \sim 1 \times 10^{-21}\left(\frac{E}{10^{-6} M_{\odot} c^{2}}\right)^{1 / 2}\left(\frac{10 \mathrm{kHz}}{f}\right)^{1 / 2}\left(\frac{50 \mathrm{kpc}}{r}\right),
$$

for the fundamental $w$-mode. Here we have used typical parameters for the pulsation modes, and the distance scale used is that to SN1987A. In this volume of space one would not expect to see more than one event per ten years or so. However, the assumption that the energy release in gravitational waves in a supernova is of the order of $10^{-6} M_{\odot} c^{2}$ is very conservative [26].

An important factor for the detection of gravitational waves is the pulsation mode frequencies. Existing resonant gravitational wave detectors, as well as the laser interferometric ones which are under construction, are only sensitive in a certain bandwidth. The bar detector are typically tuned to $1-3 \mathrm{kHz}$, while the interferometers are sensitive within 10-1000 Hz. The initial burst-like part of the waveform which carries away whatever deformation a collapse left in the spacetime is expected to be in the frequency range of 8-10 $\mathrm{kHz}$. This is well away from the sensitivity of any resonant detector in operation. The sinusoidal part of the waveform which follows will be in the frequency range of $1.5-6 \mathrm{kHz}$ 
(for the fundamental and the first $p$-mode of the star). These pulsations are consequently well suited for detection by the existing resonant detectors.

3. Stellar pulsations: the theoretical minimum. For the study of stellar oscillations we shall consider a spherically symmetric static spacetime which can be described by the Schwarzschild solution both inside and outside the star. If we assume a small variation in the fluid or/and in the spacetime we must deal with the perturbed Einstein equations

$$
\delta\left(G_{\nu}^{\mu}-\frac{8 \pi G}{c^{4}} T_{\nu}^{\mu}\right)=0
$$

and the variation of the conservation of the mass and the momentum of the fluid

$$
\delta\left(T_{\nu ; \mu}^{\mu}\right)=0
$$

The perturbed metric will be

$$
g_{\mu \nu}=g_{\mu \nu}^{0}+h_{\mu \nu}
$$

where $g^{0}{ }_{\mu \nu}$ is the unperturbed metric and $h_{\mu \nu}<<1$. By analyzing the above system of equations we can rewrite the whole problem as two coupled wave equations, which symbolically can be written:

$$
\begin{aligned}
& \left(\frac{1}{C_{s}^{2}} \frac{\partial^{2}}{\partial t^{2}}-\frac{\partial^{2}}{\partial r_{*}^{2}}\right) W+F\left(W^{\prime}, W, Z^{\prime}, Z ; \ell\right)=0 \\
& \left(\frac{1}{c^{2}} \frac{\partial^{2}}{\partial t^{2}}-\frac{\partial^{2}}{\partial r_{*}^{2}}\right) Z+G\left(W^{\prime}, W, Z^{\prime}, Z ; \ell\right)=0 .
\end{aligned}
$$

Here $C_{s}$ is the sound speed and $r_{*}$ the tortoise radial coordinate. To analyze these two equations we assume a harmonic time dependence $Z, W \approx \exp i \omega t$. We also need to introduce suitable boundary conditions. These are (i) regular behaviour of all functions at the center of the star, (ii) vanishing of the lagrangian variation of the pressure at the surface and (iii) no incoming waves from infinity. This then formulates an eigenvalue problem, in which the eigenvalues are the frequencies $\omega$ of the oscillation. Each of the two wave equations describes the motion of one of the two components of the system, the fluid and the spacetime. The first equation describes the fluid perturbation. That is, $W$ can be $\delta p$ or $\delta \rho$ ( $\delta$ stands for lagrangian variation), or one of the components of the fluid displacement vector, or any combination of these quantities. The second equation describes the perturbation of the spacetime. In this case $Z$ can be either $h_{t t}$ or $h_{\theta \theta}$ or a combination of them. Thus we expect that equation (7) will describe mainly the fluid oscillations while (8) corresponds to the spacetime ones. This can easily be seen in two limiting cases. Let us first assume that the gravitational field is very weak. Then equation (8) can be omitted and we can find that one equation is enough to describe (with acceptable accuracy) the oscillations of the fluid (Cowling approximation [12]). In the alternative case we assume that the coupling between the two equations is weak and can consequently derive all the features of the spacetime perturbations from equation (8) (Inverse Cowling Approximation [5]).

The decomposition of the above system of equations into tensor spherical harmonics leads to two classes of oscillations according to the parity of the harmonics. The first 
ones called even (or spheroidal or polar) produce spheroidal deformations on the fluid, while the second are the odd (or toroidal or axial) which produce toroidal deformations. If rotation and shear stresses are absent, the odd parity oscillations are degenerate in the Newtonian limit. In the relativistic case they don't produce any fluid deformations at all. Instead they produce a non-varying continuous rotation in the fluid.

4. Normal mode analysis. The study of stellar oscillations in a general relativistic context has already a history of 30 years. Nevertheless, recent results have shown remarkable features which had previously been overlooked. Until recently most studies treated the stellar oscillations in a nearly Newtonian manner, thus practically ignoring the dynamical properties of the spacetime. The spacetime was used as the medium upon which the gravitational waves produced by the oscillating star propagate.

Inspired by a simple but instructive model [21] Kokkotas and Schutz [22] showed the existence of a new family of modes: the $w$-modes. These are clearly spacetime modes and their properties, althought different, are closer to the black-hole normal modes than to the standard fluid stellar modes. The main characteristics of the $w$-modes are high frequencies accompanied with very rapid damping. Furthermore, these modes hardly excite any fluid motion. The existence of these modes has been verified by subsequent work [23], [4] and it has been shown that they exist also for axial oscillations [20]. Moreover, subfamilies of $w$-modes have been found for both the polar and axial oscillations i.e. the interface [23], [3] and the trapped modes [11], [19], [3]. Recently, it has been proven that one can reveal all the properties of the $w$-modes even if one "freezes" the fluid oscillations (Inverse Cowling Aproximation) [5]. In the rest of this section we shall describe the features of both families of oscillation modes.

4.1. Families of fluid modes. The fluid modes exist only for even parity oscillations. Here we will describe the properties of the most important modes for gravitational wave emission. These are the fundamental, the pressure and the gravity modes. For an extensive discussion of other families of fluid modes we refer the reader to [17]

- The $f$-mode (fundamental) is a stable mode which exists only for non-radial oscillations and describes surface waves. The frequency is proportional to the mean density of the star and it is nearly independent of the details of the stellar structure. Its eigenfunctions have no nodes inside the star, and they grow towards the surface. The $f$-mode is due to the interface between the star and its surroundings. A typical neutron star has an $f$-mode with a frequency of $1.5-3 \mathrm{kHz}$ and the damping time of this oscillation is less than a second.

- The $p$-modes (pressure or acoustic) exist for both radial and non-radial oscillations. There are infinitely many of them. The pressure is the restoring force and it experiences substantial fluctuations when these modes are excited. Usually, the radial component of the fluid displacement vector is significantly larger than the tangential component. The oscillations are thus nearly radial. The frequencies depend on the travel time of an acoustic waves across the star. For a neutron star the frequencies are typically higher than $4-5 \mathrm{kHz}\left(p_{1}\right.$-mode) and the damping times are of the order of a few seconds (they increase with the order of the mode). 
- The $g$-modes (gravity) arise because gravity tends to smooth out material inhomogeneities along level-surfaces with $U=$ constant. The $g$-modes need non-zero temperature to exist, and if they exist there are infinitely many of them. These modes correspond to very small changes in pressure along the star. Usually, the tangential components of the fluid displacement vector are dominant in the fluid motion. The $g$-modes are all degenerate at zero frequency for a star in convection and they can be unstable. For typical neutron stars they have frequencies smaller than a few hundred $\mathrm{Hz}$ (the frequency decreases with the order of the mode), and they usually damp out in time longer than a few seconds. The $g$-mode oscillations can, in fact, even last a few minutes.

4.2. Families of spacetime or w-modes. The three known families of $w$-modes have exactly the same behaviour both for even and odd parity stellar oscillations.

- The curvature modes are the standard $w$-modes. They are the most important for astrophysical applications [22]. They are clearly related to the spacetime curvature [2] and exist for all relativistic stars. Their main characteristic is the rapid damping of the oscillations. The damping rate increases as the compactness of the star decreases: For nearly Newtonian stars these modes cannot be calculated numerically. One of their main characteristics is the absence of significant fluid motion (this is a common feature for all families of $w$-modes). Numerical studies have shown the existence of an infinite number of modes. For a typical neutron star the frequency of the first $w$-mode is around $8-12 \mathrm{kHz}$ and it increases with the order of the mode. Meanwhile the typical damping time is of the order of a few tenths of a millisecond and decreases slowly with the order of the mode.

- The trapped modes, exist only for supercompact stars $(R / M \leq 3)$ i.e. when the surface of the star is inside the peak of the gravitational field's potential barrier [11],[20]. They do not induce any significant fluid motions and there is only a finite number of them (usually less than seven or so). The number of trapped modes increases as the potential well becomes deeper, i.e. with increasing compactness of the star. Their damping is quite slow since the gravitational waves have to penetrate the potential barrier. Their frequencies can be of the order of a few hundred $\mathrm{Hz}$ to a few $\mathrm{kHz}$ while their damping times can be of the order of a few tenths of a second. In general no realistic equations of state are known that would allow the formation of a sufficiently compact star for the trapped modes to be relevant.

- The interface modes [23] are extremely rapidly damped modes. It seems that there is only a finite number of such modes (2-3 modes only) [3]. They are in some ways similar to the modes for acoustic waves scattered of a hard sphere. They do not induce any significant fluid motion and their frequencies can be from 6 to 15 $\mathrm{kHz}$ for typical neutron stars.

5. Estimation of the stellar parameters. For astronomy it is important not only to observe various astronomical phenomena but also to try to mine information from these observations. From the observations of solar and stellar oscillations (normal stars) 


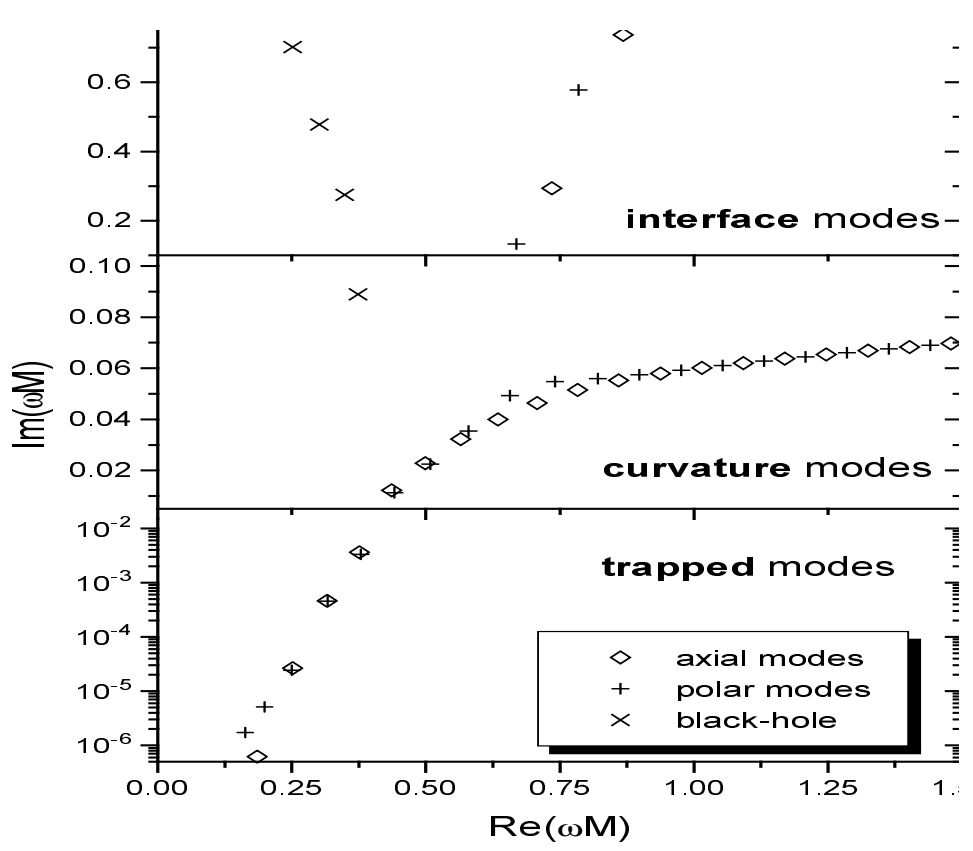

Figure 1: A graph which shows all the $w$-modes: curvature, trapped and interface both for axial and polar perturbations for a very compact uniform density star with $M / R=0.44$. The black-hole spectrum is also drawn for comparison. As the star becomes less compact the number of trapped modes decreases and for a typical neutron star $(M / R=0.2)$ they disappear. The $\operatorname{Im}(\omega)=1 /$ damping of the curvature modes increases with decreasing compactness and for a typical neutron star the first curvature mode nearly coincides with the fundamental blackhole mode. The behaviour of the interface modes changes slightly with the compactness. The similarity of the axial and polar spectra is apparent.

astronomers have managed to get details of the internal structure of the star. Now the GONG program [18] for detailed observation of the solar seismology is well underway. These ideas have prompted us to examine the possibility of getting information about the neutron star parameters (mass, radius) and internal structure from possible detections of gravitational waves from a pulsating neutron star.

It will be instructive to briefly examine the case of oscillating black holes since they are much "cleaner" objects than stars. From the normal mode analysis of black hole oscillations we can get a spectrum which is related to the parameters of the black hole (mass and angular momentum) using the following empirical relations $[14,15]$ for the frequency

$$
\omega=\frac{1}{2 \pi M}\left[1-\frac{63}{100}(1-a)^{3 / 10}\right]
$$

and the damping time of the oscillations

$$
\tau=\frac{4 M}{(1-a)^{9 / 10}}\left[1-\frac{63}{100}(1-a)^{3 / 10}\right]^{-1}
$$


Here $M$ is the black hole mass and $a$ is the angular momentum (in geometrical units). We should point out here that only the fundamental mode of oscillation contributes; the high-order black-hole modes are insignificant because of their extremely rapid damping.

A similar set of empirical relations cannot be derived in the case of neutron star oscillations since the stars are not as "clean" as black holes. For example there is no unique way of connecting the mass with the radius since the details of the stellar structure are involved. Additionally, more than one frequency contributes. Although we expect that most of the dynamical energy stored in the fluid oscillations will be radiated away in the $f$-mode, some of the $p$-modes may be excited as well and a significant amount of energy could be radiated away through these modes [1]. As far as the spacetime modes are concerned we expect that only the curvature modes (the standard $w$-modes) will be excited but it is possible that the radiated energy can be shared between the first 2-3 $w$-modes [1].

However, it is possible to derive relations similar to (9) and (10) for the frequencies and the damping times of the various families of modes. From such relations (since we will have more than two of them) we can put strong constraints on the equation of state.

In order to get such relations one must calculate the normal mode frequencies for the fluid and spacetime modes for different equations of state. But in order to get the correct relation between the stellar parameters and the frequencies one should understand the nature of each family of modes and normalize them accordingly. In the following we shall give some hints to how this can be done.

As has been mentioned earlier the characteristic pulsation time of the $f$-mode is proportional to the mean density of the star. This is clear from the following qualitative relations

$$
T_{f} \sim \frac{R}{\bar{v}} \sim \frac{R}{(M / R)^{1 / 2}} \sim\left(\frac{R^{3}}{M}\right)^{1 / 2} \sim \frac{1}{\bar{\rho}^{1 / 2}}
$$

where $\bar{v}$ is the average speed of sound. In the same way we can get for the damping time of the $f$-mode that

$$
\tau_{f} \sim \frac{L_{i n t}}{L_{G W}} T_{f} \sim \frac{T_{f}}{L_{i n t}} \sim \frac{T_{f}}{E_{k i n} / T_{f}} \sim\left(\frac{R}{M}\right)^{3} R
$$

where $L_{i n t}$ is the internal power flow and $L_{G W}$ the power output in gravitational waves $[25]$.

For the frequency of the $w$-modes we do not have any similar relation. We can only say that the order of the mode is inversely proportional to the radius of the star

$$
\omega_{w} \sim\left(n+\frac{1}{2}\right) \frac{\pi}{R}
$$

But although this is not very encouraging it can be useful in identifying the stellar equation of state.

Finally, a very useful relation can be derived from the observation that the damping times of the $w$-modes are proportional to the compactness of the star i.e.

$$
\tau_{w} \sim \frac{M}{R}
$$


Table 1

Using the relations (15), (17), and (16) we estimate the masses and the radii for polytropic stars with $N=0.8,1,1.2$. The errors of these estimates are shown. The notation $\omega_{f}-\tau_{w}$ (etcetera) means that we use the frequency of the $f$-mode, eqn (15), and the damping of the $w$-mode, eqn (16), to approximate the stellar parameters.

\begin{tabular}{|c|ccc|ccc|}
\hline $\mathrm{N}$ & $\begin{array}{c}R \\
(\mathrm{Km})\end{array}$ & $\begin{array}{c}\omega_{f}-\tau_{w} \\
\text { error } \%\end{array}$ & $\begin{array}{c}\omega_{f}-\tau_{f} \\
\text { error } \%\end{array}$ & $M / M_{\odot}$ & $\begin{array}{c}\omega_{f}-\tau_{w} \\
\text { error } \%\end{array}$ & $\begin{array}{c}\omega_{f}-\tau_{f} \\
\text { error } \%\end{array}$ \\
\hline 0.8 & 10.026 & .96 & .95 & 1.084 & 6.30 & .76 \\
0.8 & 9.493 & 1.02 & 1.08 & 1.357 & 1.46 & 7.46 \\
1.0 & 8.863 & 5.98 & 7.72 & 1.266 & 8.72 & 13.69 \\
1.0 & 7.415 & .99 & .31 & 1.351 & .43 & 1.63 \\
1.2 & 12.771 & 4.34 & 5.10 & 1.237 & 4.26 & 1.80 \\
1.2 & 8.968 & 5.18 & 3.46 & 1.459 & 1.54 & 7.16 \\
\hline
\end{tabular}

Using data for a set of stars with seven different equations of state, we have derived several empirical relations. The compactness $M / R$ of the considered stars lies in the interval $0.15-0.30$. For the $f$-mode frequency we find that

$$
\omega_{f}(\mathrm{kHz}) \approx 0.247+2.195\left(\frac{\bar{M}}{\bar{R}^{3}}\right)^{1 / 2},
$$

while the damping rate of the $w$-mode behaves as ( $\tau_{\omega}$ is the e-folding time of the pulsation)

$$
\frac{1}{\tau_{w}}(\mathrm{MHz}) \approx 103.7-63.5\left(\frac{\bar{M}}{\bar{R}}\right)
$$

Finally, the $f$-mode damping is

$$
\frac{1}{\tau_{f}}(\mathrm{~Hz})=\left(\frac{\bar{M}^{3}}{\bar{R}^{4}}\right)\left[31.63-31.28\left(\frac{\bar{M}}{\bar{R}}\right)+7.58\left(\frac{\bar{M}}{\bar{R}}\right)^{2}\right]
$$

where $\bar{M}=M /\left(1.4 M_{\odot}\right)$ and $\bar{R}=R /(10 \mathrm{~km})$.

This idea seems promising and is simple enough, but will it be useful in practice? Let us give an example for stars with polytropic equations of state: We have constructed a set of independent polytropic stellar models $\left(p=K \rho^{1+1 / N}\right)$ with varying polytropic index $(N=0.8 ; 1 ; 1.2)$. We have determined the $f$-mode and the slowest damped polar $w$-mode for each of these models. This data represents the "observed" gravitational-wave signal. Then we used the empirical relations (15), (17) and (16) to estimate the mass and radius of each star. Finally, the resulting values were compared to the true ones. The results of this comparison - shown in Table 1- clearly demonstrate the robustness of the empirical relations: The error is usually smaller than $10 \%$.

The presented results provide the first step towards a full discussion of this problem. Much future work is required before definite conclusion about the usefulness of the proposed scheme can be drawn. It is important to incorporate the estimated effect of statistical and measurement errors in the analysis. Early results [7] show that these effects add an error in the calculation of radius and mass of the star which is less than a few percent for 

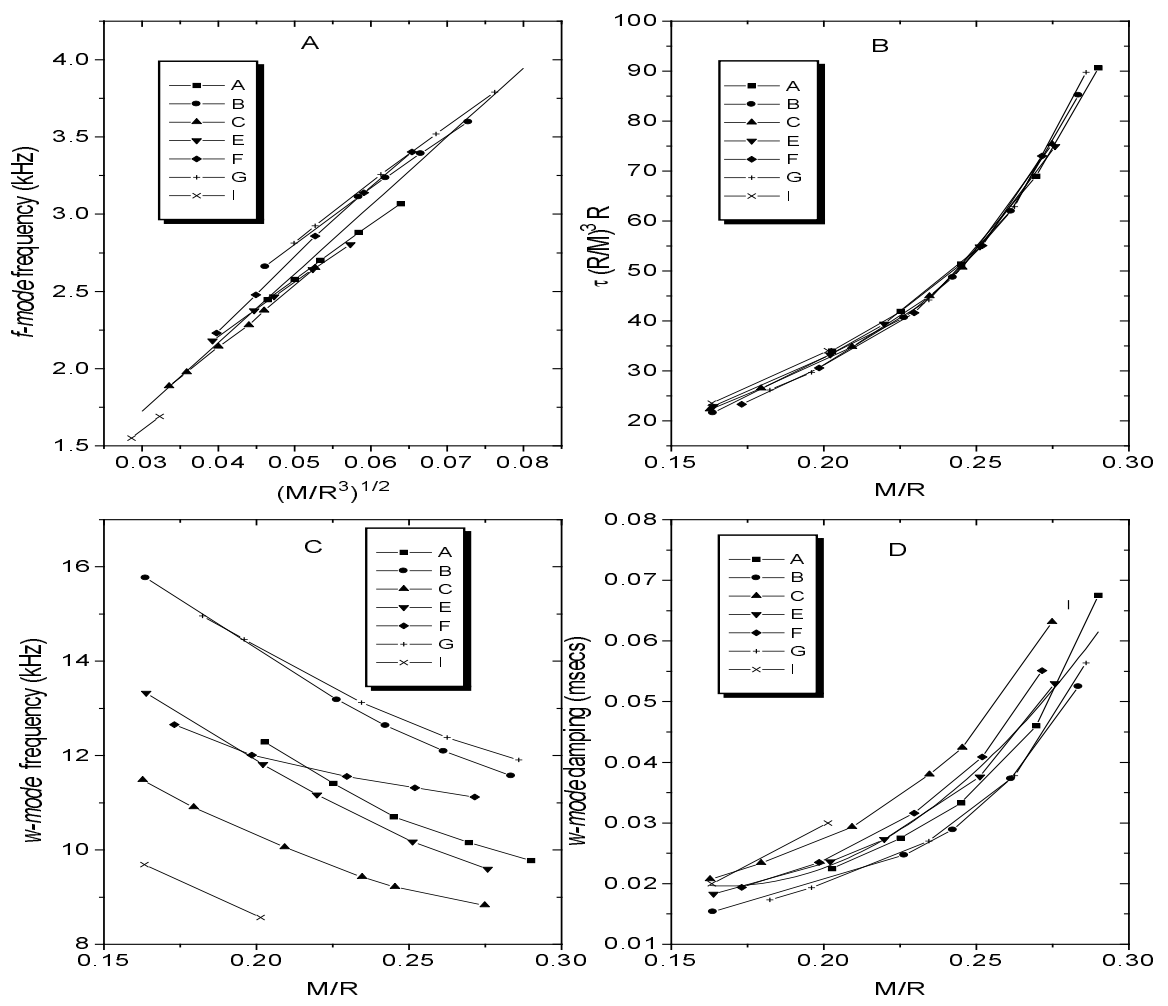

Figure 2: Data for 33 stellar models and seven equations of state (as defined in [24]) are plotted. We show (A) the pulsation frequency of the $f$-mode as a function of the average density, (B) the e-folding time for the $f$-mode as a function of the stellar compactness, $(\mathrm{C})$ the frequency of the $w$-mode as function of the stellar compactness, (D) the e-folding time for the slowest damped $w$-mode as a function of the stellar compactness (dimensionless).

stars in our galaxy. For this work to be complete it is important to obtain fits similar to (15), (17) and (16) for a larger number of realistic EOS. Nevertheless, it seems likely that the general principle discussed here will not be significantly changed by the inclusion of data from further equations of state. Moreover, if the star is rotating the mode-spectrum changes and the degree of deviation from the non-rotational case can then help us estimate the rotation rate of the star just as for the black-hole case [15]. This is an interesting prospect for the future.

6. Exciting the stellar modes. The discussion in the previous sections has shown that we have an acceptable knowledge of stellar pulsations and that we can extract information from the detection of such oscillations. But as was clear from the discussion in section 2, the energy released as gravitational radiation during the stellar collapse is basically unknown. Additionally, it is not known how the energy is distributed in the various fluid and spacetime modes. Both uncertainties depend stongly on the details of gravitational collapse, or in general the mechanism that excites the modes. Unless full 3D general relativistic codes are generated for the gravitational collapse or the final 
stages of binary coalescence we will never be able to give definite answers to the above questions. In the meantime, perturbation theory is a reliable way to get some first hints and indications. It should certainly be able to tell us which of the modes can be excited.

Recently, Andersson and Kokkotas [6] have studied the excitation of odd parity or axial modes by sending gravitational wave pulses to hit the star. The results of this study are encouraging because they provide the first indication that the $w$-modes can be excited in a dynamical scenario. Similar results have recently been obtained by Borelli and Ferrari [9]. They study the excitation of the axial modes due to particles falling towards the star.

These results have prompted the study of an astrophysically more relevant problem; the excitation of even parity (or polar) stellar oscillations. In recent work Allen et al [1] have studied the excitation of the polar modes using two sets of initial data. First, as previously for the axial case, they excited the modes by an incoming gravitational wave pulse. In the second case, the initial data described an initial deformation of both the fluid and the spacetime. In the first case the picture was similar to that of the axial modes i.e. the star was excited and emitted gravitational waves in both $w$ and the fluid modes. Nearly all of the energy was radiated away in the $w$-mode frequencies. This should be expected since the incoming pulse does not have enough time to couple with the fluid (which has a much lower speed of propagation of information). The infalling gravitational waves are simply affected by the spacetime curvature associated with the star, and the outgoing radiation contains an unmistakable $w$-mode signature. In the second case one has considerable freedom in choosing the degree of initial excitation of the fluid and the spacetime. In the study the choice was some "logical" initial data inspired by the treatment of the problem in the Newtonian limit. Then the energy emitted as gravitational waves was more evenly shared between the fluid and the spacetime modes, and one could see the $f, p$ and $w$ modes in the signal. The characteristic signal was (as expected) a short burst ( $w$-modes) followed by a slowly damped sinusoidal wave (fluid modes).

These new results show the importance of general relativity for the calculation of the energy emission in violent processes. Up to now the general way of calculating the energy emission has been the quadrupole formula. But it only accounts for the fluid deformations and motions, and as we have seen this accounts only for part of the total energy.

7. Conclusions. From the previous discussion we can draw some general conclusions and suggestions for future work

7.1. From Perturbation Theory we will expect to (i) complete the statistics and to improve the empirical relation including all available EOS. This will be of great importance if we will ever be in a position to analyze gravitational-wave signals from neutron stars. (ii) to include in the empirical relations the effects of rotation.

7.2. From Numerical Relativity we will expect (i) to enhance the results of [1] by providing initial data for stellar collapse in ways similar to the pioneering work of $[13,27$, $28,29]$. (ii) to produce 3D general relativistic codes for both the stellar collapse and the final stages of binary coalescence (iii) to explain in detail the observed high velocities of pulsars, the conditions under which these high velocities have been achieved during the collapse and the way that they are connected to the gravitational wave emission. 
7.3. The Detectors have to be improved. Specifically they must become sensitive in a larger bandwidth: Most present detectors operate in the $1-3 \mathrm{kHz}$ regime while for example the $w$-modes are in the $8-12 \mathrm{kHz}$ regime [16]. We also need detectors to cover the whole bandwidth from $1-10 \mathrm{kHz}$ i.e. a gravity Xylophone.

I thank G. Allen and N. Andersson for useful comments and discussions. The financial support from Max-Planck is greatfully acknowledged. This work was supported by an exchange program from the British Council and the Greek GSRT.

\section{References}

[1] G. D. Allen, N. Andersson, K. D. Kokkotas and B. F. Schutz, in preparation (1996).

[2] N. Andersson, Gen. Rel. Grav. in press (1996).

[3] N. Andersson, Y. Kojima and K. D. Kokkotas, Ap. J. 462855 (1996).

[4] N. Andersson, K. D. Kokkotas and B. F. Schutz, MNRAS 2741039 (1995).

[5] N. Andersson, K. D. Kokkotas and B. F. Schutz, MNRAS 2801230 (1996).

[6] N. Andersson and K. D. Kokkotas, Phys. Rev. Lett. in press (1996).

[7] T. Apostolatos and K. D. Kokkotas, preprint (1996).

[8] S. Bonazzola and J.-A. Marck, in Relativity in General, Eds J. D. Alonso and M. L. Paramo, Editions Frontieres, Gif-sur-Yvette (1994) p. 15.

[9] A. Borelli and V. Ferrari, preprint (1996).

[10] A. Burrows and J. Hayes, Phys. Rev. Lett. 76352 (1996).

[11] S. Chandrasekhar and V. Ferrari, Proc. R. Soc. Lond. A 434, 449 (1991).

[12] T. G. Cowling, M.N.R.A.S. 101367 (1941).

[13] C. T. Cunningham, R. H. Price and V. Moncrief, Astrophys. J 224, 643 (1978); Astrophys. J 230, 870 (1979); Astrophys. J 236, 674 (1980).

[14] F. Echeverria, Phys. Rev. D 40, 3194 (1989).

[15] L. S. Finn, Phys. Rev. D 46, 5236 (1992).

[16] S. Frasca and M. A. Papa, Int. J. Mod. Phys. 41 (1995).

[17] A. Gautschy and H. Saio, Annu. Rev. Astron. Astrophys. 3375 (1995).

[18] J. W. Harvey et al., Science 2721284 (1996).

[19] Y. Kojima, N. Andersson and K. D. Kokkotas, Proc. R. Soc. Lond. A 451341 (1995).

[20] K. D. Kokkotas, MNRAS 2681015 (1994), Erratum: 2771599 (1995).

[21] K. D. Kokkotas and B. F. Schutz, Gen. Rel. Grav. 18913 (1986).

[22] K. D. Kokkotas and B. F. Schutz, MNRAS 255119 (1992).

[23] M. Leins, H.-P. Nollert and M. H. Soffel, Phys. Rev. D 483467 (1993).

[24] L. Lindblom and S. L. Detweiler, Ap. J. Suppl. 5373 (1983).

[25] C. W. Misner, K. S. Thorne and J. A. Wheeler, Gravitation, W.H. Freeman (1973).

[26] B. F. Schutz, Detection of gravitational waves, in Proceedings of "Astrophysical sources of gravitational radiation" Eds: J. A. Marck and J. P. Lasota (Cambridge University Press, 1996).

[27] E. Seidel and T. Moore, Phys. Rev. D 352287 (1987).

[28] E. Seidel, E. S. Myra and T. Moore, Phys. Rev. D 382349 (1988).

[29] E. Seidel, Phys. Rev. D 421884 (1990); 44950 (1991).

[30] S. van den Bergh and G. A. Tammann, Annu. Rev. Astron. Astrophys. 29363 (1991). 